\title{
BEYOND THE BULGE: A FUNDAMENTAL RELATION BETWEEN SUPERMASSIVE BLACK HOLES AND DARK MATTER HALOS
}

\author{
LAURA FERRARESE \\ Rutgers University, New Brunswick, NJ, 08854, lff@physics.rutgers.edu \\ The Astrophysical Journal
}

\begin{abstract}
The possibility that the masses $M_{\bullet}$ of supermassive black holes (SBHs) correlate with the total gravitational mass of their host galaxy, or the mass $M_{D M}$ of the dark matter halo in which they presumably formed, is investigated using a sample of 16 spiral and 20 elliptical galaxies. The bulge velocity dispersion $\sigma_{c}$, typically defined within an aperture of size $R \lesssim 0.5 \mathrm{kpc}$, is found to correlate tightly with the galaxy's circular velocity $V_{c}$, the latter measured at distances from the galactic center at which the rotation curve is flat, $R \sim 20-80 \mathrm{kpc}$. By using the well known $M_{\bullet}-\sigma_{c}$ relation for SBHs, and a prescription to relate $v_{c}$ to the mass of the dark matter halo $M_{D M}$ in a standard $\Lambda \mathrm{CDM}$ cosmology, the correlation between $\sigma_{c}$ and $v_{c}$ is equivalent to one between $M_{\bullet}$ and $M_{D M}$. Such a correlation is found to be nonlinear, with the ratio $M_{\bullet} / M_{D M}$ decreasing from $2 \times 10^{-4}$ for $M_{D M} \sim 10^{14} M_{\odot}$, to $10^{-5}$ for $M_{D M} \sim 10^{12} M_{\odot}$. Preliminary evidence suggests that halos of mass smaller than $\sim 5 \times 10^{11} M_{\odot}$ are increasingly less efficient - perhaps unable - at forming SBHs.
\end{abstract}

\section{INTRODUCTION}

Never has the study of supermassive black holes (SBHs) been greeted with such attentiveness as in the past few years. Although by the mid 1960s it was generally recognized that the energy source of the newly discovered quasars must be gravitational (e.g. Robinson 1965), for the following three decades the existence of SBHs was destined to be surrounded by skepticism. By the mid 1990s, a few years after the launch of the Hubble Space Telescope, it was widely accepted (Kormendy \& Richstone 1995). Today, SBHs feature prominently in models for the formation and evolution of their host galaxies (e.g. Silk \& Rees 1998). Freed from the burden of having to demonstrate the very existence of SBHs, we can now begin asking cardinal questions: how did SBHs form, how do they accrete, how do they evolve, and what role do they play in the formation of cosmic structure?

Tackling such questions has been made possible by the discovery that SBHs are tightly related to the large-scale properties of their host galaxies. Black holes masses, $M_{\bullet}$, correlate with the blue luminosity $L_{B}$ of the host bulge (Kormendy \& Richstone 1995). Even more compelling is the correlation between $M_{\bullet}$ and the bulge velocity dispersion, $M_{\bullet} \propto \sigma^{\alpha}$ (Ferrarese \& Merritt 2000; Gebhardt et al. 2000). Using the most up-todate set of SBH mass measurements such a correlation can be written as:

$$
M_{\bullet}=(1.66 \pm 0.32) \times 10^{8}\left(\sigma_{c} / 200 \mathrm{~km} \mathrm{~s}^{-1}\right)^{4.58 \pm 0.52} M_{\odot}
$$

(Ferrarese 2002), where $\sigma_{c}$ is normalized to an aperture of size one eighth the bulge effective radius. The $M_{\bullet}-\sigma_{c}$ relation has supplanted the $M_{\bullet}-L_{B}$ relation as the tool of choice for the study of SBH demographics (Ferrarese 2002; Yu \& Tremaine 2002), for the following reasons. The $M_{\bullet}-L_{B}$ relation suffers from a large scatter (a factor of several in $M_{\bullet}$, Ferrarese \& Merritt 2000), most (but not all) of which is ascribable to lenticular and spiral galaxies (McLure \& Dunlop 2002). The $M_{\bullet}-\sigma_{c}$ relation, on the other hand, appears to be intrinsically perfect (Ferrarese \& Merritt 2000; Merritt \& Ferrarese 2001a; Ferrarese 2002). It holds true for galaxies of disparate Hubble types (from
SBs to compact ellipticals to $\mathrm{cDs}$ ) belonging to wildly different environments (from rich clusters to the field), showing perfectly smooth (e.g. NGC 6251) or highly disturbed (e.g. NGC 5128) morphologies. Furthermore, $\sigma$ is a more faithful gauge of mass than $L_{B}$ : the $M_{\bullet}-\sigma_{c}$ relation thus betrays the existence of a tight connection between the masses of SBHs and those of the hot stellar component surrounding them (Merritt \& Ferrarese 2001b).

Have we indeed found, in the $M_{\bullet}-\sigma_{c}$ relation, the fossil record that links the formation and evolution of SBHs to those of their host galaxies? The $M_{\bullet}-\sigma_{c}$ relation, and the equally tight relation recently discovered by Graham et al. (2001) between $M_{\bullet}$ and the central concentration of bulge light, probe the connection between SBHs and the baryonic component of galactic bulges. Measurements of $\sigma$ typically do not extend far enough to penetrate the region dominated by the dark matter (DM) component (Faber \& Gallagher 1979). And yet, it is not the mass of the bulge but rather, the total mass $M_{t o t}$ of the galaxy or the mass $M_{D M}$ of the DM halo, which is the key ingredient in most theoretical models proposed for the formation of SBHs (Adams, Graff \& Richstone 2000; Monaco et al. 2000; Haehnelt, Natarajan \& Rees 1998; Silk \& Rees 1998; Haehnelt \& Kauffmann 2000; Cattaneo, Haehnelt \& Rees 1999; Loeb \& Rasio 1994). Once a correlation with $M_{t o t}$ (or $M_{D M}$ ) is recovered by the models, the correlation with bulge mass is implicit because, in standard CDM scenarios, the bulge mass is loosely determined by the halo properties (e.g. van den Bosch 2000; Haehnelt, Natarajan \& Rees 1998; Zhang \& Wyse 2000).

This paper pursues, from an observational standpoint, the possibility of a link between $M_{\bullet}, M_{t o t}$ and $M_{D M}$. A tight correlation is indeed found in the form of a relation between $M_{\bullet}$ and the circular velocity of the SBH host galaxy, measured well beyond the optical radius.

\section{DATABASE}

Measuring the total gravitational mass of a galaxy is a formidable challenge. In bright ellipticals, the temperature and density distribution of the thermal X-ray gas can lead to a measure of $M_{t o t}$ under the minimal condition of hydrostatic equilib- 
while much progress is expected as Chandra data are collected,

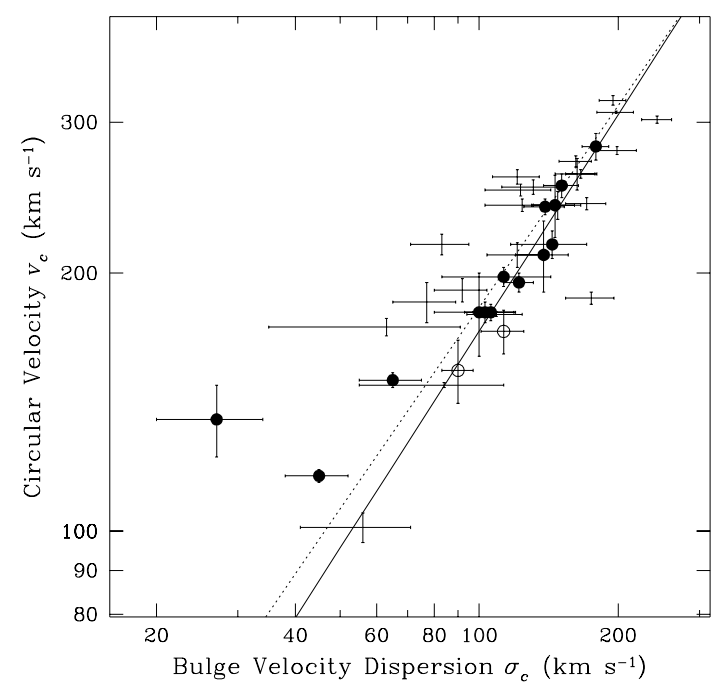

FIG. 1.-Correlation between bulge velocity dispersion $\sigma_{c}$ and disk circular velocity $v_{c}$ for a sample of 37 galaxies with either optical (open circles) or $\mathrm{HI}$ (solid circles) rotation curves. Points marked by errorbars only correspond to galaxies for which the rotation curves does not extend beyond $R_{25}$. The galaxy to the far left, with the smallest value of $\sigma_{c}$, is NGC 598. The solid line corresponds to a fit to all galaxies with $\sigma_{c}>70 \mathrm{~km} \mathrm{~s}^{-1}$ and $R\left(v_{c}\right) / R_{25}>1.0$. The dotted line corresponds to the fit to all galaxies, with the only exclusion of NGC 598.

previous generations of X-ray satellites lacked the necessary combination of spectral response and sensitivity to allow an accurate measurement of all required observables (e.g. Biermann et al. 1989; Awaki et al. 1994; Matsushita et al. 1998). Stars, globular cluster and planetary nebulae (PNe) are good tracers of the gravitational potential (e.g. Saglia et al. 2000; Côté et al. 2001; Arnaboldi et al. 1998). However, the derived masses suffer large uncertainties due to the unknown velocity anisotropy of the system and, in particular for the case of PNe, the small sample size of the tracers (Napolitano et al. 2001; Merritt \& Tremblay 1993).

In spiral galaxies, however, the rotation curves of the cold disk component bear the direct imprint of the mass distribution. Indeed, the lack of a decline in the circular velocity $v_{c}$ at radii of order and beyond the optical radius of spirals was one of the first indications of the existence of a significant dark matter component (Rubin, Ford \& Thonnard 1978, 1980; Rubin, Thonnard \& Ford 1977; Krumm \& Salpeter 1979). To first order, therefore, a relation between $M_{\bullet}$ and $M_{t o t}$ would be reflected in a relation between $M_{\bullet}$ and $v_{c}$.

Of the four spiral galaxies with a secure measurement of $M$ • (see the compilation by Merritt \& Ferrarese 2002) only two, the Milky Way and NGC 4258, have published rotation curves, both derived from 21-cm studies (Olling \& Merrifield 1998; van Albada 1980). However, the sample can be augmented if one is willing to exchange $M_{\bullet}$ for the bulge velocity dispersion $\sigma_{c}$, following equation (1). Of all spirals for which $\sigma$ has been measured, 46 have rotation curves, either in HI (21 objects) or optical ( 25 objects). For six of the galaxies with an HI rotation curve, warnings are found in the original papers as to the use of the published circular velocities for mass measurements. Reasons include warps in the HI disk preventing one from deprojecting the observed rotation curve (NGC 4565, Rupen 1991;
NGC 891, Rogstad \& Shostak 1972; NGC 1808, Saika et al.

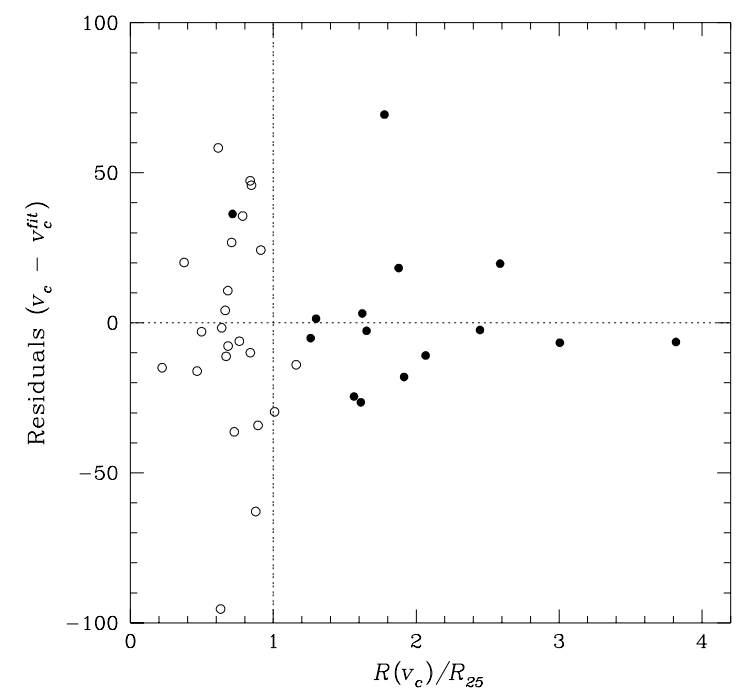

FIG. 2.- Residuals from the best fit to all galaxies with the exclusion of NGC 598 (dotted line in Fig. 1), plotted as a function of $R\left(v_{c}\right) / R_{25}$.

1980, Koribalski et al. 1993) and/or the presence of significant non-circular motion in the HI gas (NGC 3079, Irwin \& Seaquist 1991; NGC 3031, Rots \& Shane 1975; NGC 4736, van der Kruit 1974). For two of the galaxies with an optical rotation curve (NGC 3200 and NGC 7171), two values of $\sigma$ discrepant at the $>99.9 \%$ level exist in the literature (McElroy 1995; Whitmore et al. 1984; Dressler 1984); these galaxies have also been excluded from the sample.

Data and references for the remaining 38 galaxies are given in Table 1. To allow for the application of equation (1), the bulge velocity dispersion $\sigma$ is corrected to the equivalent of an aperture of radius $r_{e} / 8$ following the prescriptions of Jorgensen, Franx \& Kjaergaard (1995). The bulge effective radius $r_{e}$ is taken from Baggett et al. (1998, but see notes to Table 1).

\section{THE $v_{c}-\sigma_{c}$ RELATION}

Fig. 1 shows the circular velocity versus the bulge velocity dispersion within $r_{e} / 8$ for all galaxies in Table 1. The correlation is immediately apparent, but a few observations are in order.

First, a glance at Table 1 will reveal that the most noticeable difference between the two samples of optical and HI rotation curves is, not surprisingly, that while only two of the optical rotation curves extend beyond $R_{25}$, the galaxy radius at the $B=25$ mag $\operatorname{arcsec}^{-2}$ isophote, all but one of the HI rotation curves do. Inspection of the original papers shows that the HI rotation curves all exhibit a common pattern. It is well known (e.g. Sofue \& Rubin 2001 and references therein) that as one moves away from the center, $v_{c}$ rises rapidly, peaks somewhere between 3 and $15 \mathrm{kpc}$, and then remains flat (sometimes after a modest initial decline) until the last measured point, typically $R\left(v_{c}\right)=30-40 \mathrm{kpc}$. Optical rotation curves rarely extend beyond $10 \mathrm{kpc}$; at this distance $v_{c}$ has not yet settled into a flat rotation curve. In Fig. 1, galaxies with rotation curves not extending beyond $R_{25}$ (shown as errorbars only) appear to display larger scatter, relative to the mean correlation defined by all galaxies, than the galaxies with a more extended rotation 
curve.

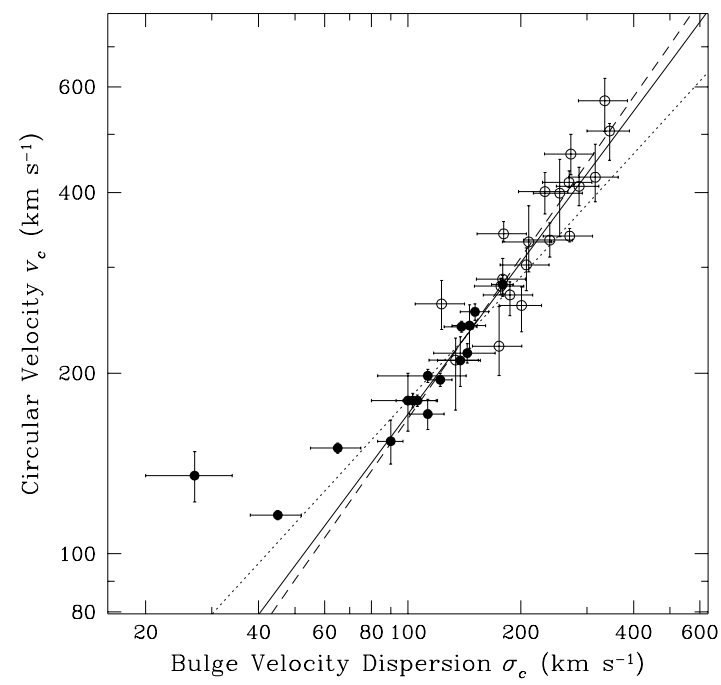

FIG. 3.- Same as Fig. 1, except solid dots represent spiral galaxies with $R\left(v_{c}\right) / R_{25}>1.0$, open dots represent the sample of elliptical galaxies from Kronawitter et al. (2000). The dotted line is a fit to all spiral galaxies with the exception of NGC 598, while the solid line is the fit to all spirals with $\sigma_{c}>70 \mathrm{~km} \mathrm{~s}^{-1}$. The latter fit is consistent with the one obtained for the elliptical galaxies alone (see fits 3 and 4 in Table 2). The dashed line is a fit to the entire sample of ellipticals plus spirals with $\sigma_{c}>70 \mathrm{~km} \mathrm{~s}^{-1}$.

Second, a simple visual inspection of Fig. 1 shows that among the galaxies with $R\left(v_{c}\right) / R_{25}>1$, NGC 598 (= M33, the galaxy with the lowest value of $\sigma_{c}$ in the plot) is a clear outlier. Furthermore, the two galaxies with $\sigma_{c}<70 \mathrm{~km} \mathrm{~s}^{-1}$ seem to have a significantly larger circular velocity that would be inferred if a linear fit (in $\log \sigma_{c}-\log V_{c}$ ) were to be performed to the rest of the sample.

To quantify the above statements, we performed a regression analysis (Akritas \& Bershady 1996) to all galaxies except NGC 598 (Table 2 and dotted line in Fig. 1). Fig. 2 shows the residuals from the best fit as a function of $R\left(v_{c}\right) / R_{25}$, confirming the larger scatter for galaxies with rotation curves not extending beyond $R_{25}$. For the scope of this paper, the usefulness of $v_{c}$ is directly related to how confidently $v_{c}$ can be used as an estimator of the total galactic mass, which in turns depends critically on the radial extent of the rotation curve. Therefore, after dutifully noting that the regression analysis produces consistent fits whether the entire sample, or just the galaxies for which $R\left(v_{c}\right) / R_{25}>1$ are used (Table 2), we will only retain the latter sample in the remainder of this paper.

Because of its apparently negligible intrinsic scatter (a reduced $\chi_{r}^{2}$ of 0.70 , Table 2) and the fact that it does not follow directly from simple dynamical arguments (see $\$ 3.1$ ), the $v_{c}-\sigma_{c}$ relation is of obvious interest. However, by targeting only spiral galaxies it lacks generality; furthermore, it samples a narrow range in $\sigma_{c}, \sim 90$ to $\sim 180 \mathrm{~km} \mathrm{~s}^{-1}$. It is interesting to compare the $v_{c}-\sigma_{c}$ relation observed for the spirals with the one derived recently by Kronawitter et al. (2000) and Gerhard et al. (2001) for a sample of 21 elliptical galaxies. For this sample, circular velocity curves are derived by applying non-parametric spherical models to the observed stellar absorption line profiles, velocity dispersion and surface brightness profiles. Kronawit- ter et al. conclude that the rotation curves resulting from the models are very likely nearly self-similar: all rotation curves are consistent with being flat outside $0.3 r_{e}$, with $r_{e}$ the galaxy's effective radius ${ }^{1}$. As pointed out by Gerhard et al. (2001), $v_{c}$ is expected to be linearly related to any other velocity scale, for instance $\sigma_{c}$, as a consequence of the near dynamical homology. The constant of proportionality is likely to depend slightly on the details of the models; Kronawitter et al. (2000) and Gerhard et al. (2001) do not discuss this point explicitely, but by comparing their results to the those obtained by van der Marel (1991) and Lauer (1985) for the same galaxies, variations of the order of a few tens of a percent are possible if different dynamical models are used.

Fig. 3 shows the $v_{c}-\sigma_{c}$ relation for the combined samples of 16 spirals with $R\left(v_{c}\right) / R_{25}>1$ (solid circles) plus the 20 ellipticals from Kronawitter et al. (2000, open circles). Values of $\sigma$ for the ellipticals are taken from Davies et al. (1987), and corrected to a common aperture size as detailed for the bulges. As expected, we recover the correlation between $v_{c}$ and $\sigma_{c}$ already reported by Gerhard et al. (2001).

The fact that the spiral and elliptical samples follow the same relation is unexpected (see §3.1) and points to a common cause connecting galaxy dynamics at small and large scales across the entire Hubble sequence. A regression analysis to the ellipticals produces a fit consistent with the one obtained for the spirals once the two galaxies with $\sigma_{c}<70 \mathrm{~km} \mathrm{~s}^{-1}$ are excluded (fits 3 and 4 in Table 2). If a linear fit is forced, $\sigma_{c}=(0.65 \pm 0.02) v_{c}$ for the ellipticals and $\sigma_{c}=(0.61 \pm 0.01) v_{c}$ for the spirals. However, because the correlation between $v_{v i r}$ and $\sigma_{c}$ for the ellipticals is partly due to the scale-free nature of the models, we use only the sample of 13 spirals with $R\left(v_{c}\right) / R_{25}>1$ and $\sigma_{c}>70 \mathrm{~km}$ $\mathrm{s}^{-1}$ in deriving the best fit to the $v_{c}-\sigma_{c}$ relation:

$$
\log v_{c}=(0.84 \pm 0.09) \log \sigma_{c}+(0.55 \pm 0.19)
$$

While this fit is strictly derived in the $70<\sigma_{c}<180 \mathrm{~km}$ $\mathrm{s}^{-1}$ range, it can also be considered valid in the $120<\sigma_{c}<350$ $\mathrm{km} \mathrm{s}^{-1}$ range populated by the ellipticals (Fig. 3 and Table 2). As is the case for the $M_{\bullet}-\sigma_{c}$ relation, the reduced $\chi_{r}^{2}=0.38$ of the fit points to a relation with negligible intrinsic scatter.

\subsection{Is the $\mathrm{v}_{c}-\sigma_{c}$ Relation a Tautology?}

The tightness of the $v_{c}-\sigma_{c}$ relation is suprising to the point of arising suspicions that we might be dealing with a tautology.

The most trivial case to consider is the following: a strict proportionality between $\sigma_{c}$ and $v_{c}$ would result if both responded to the same mass distribution. For instance, if the region within which $\sigma_{c}$ is measured extends to radii where the rotation curve is flat, $\sigma_{c}$ would naturally be linearly related to $v_{c}$ simply by virtue of the virial theorem. This is not the case for any of the galaxies considered here: even for the bulges with the largest effective radius, $\sigma_{c}$ only samples the innermost $0.5 \mathrm{kpc}$, while the rotation curve does not settle to a constant velocity until much farther out, typically 4 to $20 \mathrm{kpc}$. Fig. 4 plots the ratio of bulge central velocity dispersion to disk circular velocity against the ratio of the radii at which the two are measured (the radius at which $v_{c}$ peaks is taken as a lower limit to the radius at which the circular velocity first settles onto a flat curve). As can be seen, $R\left(v_{\max }\right) / R\left(\sigma_{c}\right)$ ranges from values of a few to over 100.

Even so, if rotation curves of spiral galaxies formed a nearly homologous family, as is the case for the elliptical galaxies of

\footnotetext{
${ }^{1}$ NGC4486B is the only galaxy for which the models are deemed unreliable, and will not be considered further.
} 
Gerhard et al. (2001), $v_{c}$ or $\sigma_{c}$ would be linearly related

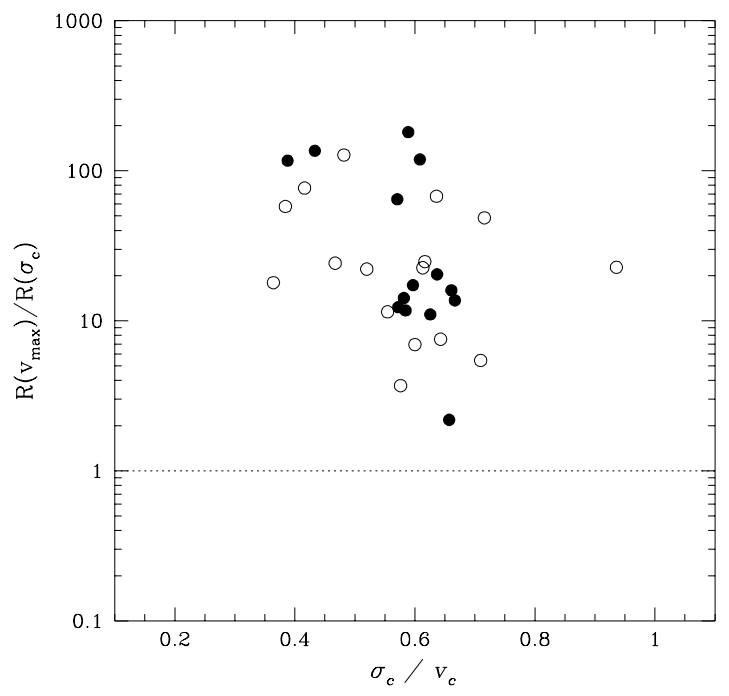

FIG. 4.- The ratio between bulge velocity dispersion $\sigma_{c}$ and disk circular velocity $v_{c}$ plotted against the ratio of the radii at which the two are measured. Solid circles are galaxies for which $R\left(v_{c}\right) / R_{25}>1.0$.

(however, the constant of proportionality would depend on the details of the mass density profile, and there would be no reason for the elliptical and spiral samples to define a common relation). Unlike Gerhard's ellipticals, however, spiral galaxies show a strong dynamical non-homology: both the profile shape and the amplitude of the rotation curves depend on the galaxy luminosity (Casertano \& van Gorkom 1991; Persic, Salucci \& Stel 1996). Therefore, a linear relation, or a relation at all, between $v_{c}$ and $\sigma_{c}$ is not expected a priori.

The two arguments presented above also explain why the $v_{c}-\sigma_{c}$ relation is not a direct result of the so called "diskhalo conspiracy". Most rotation curves are characterized by a reasonably flat rotation curve in the outer parts. This implies that the mass density profiles of the luminous and dark matter must be coupled, thereby "conspiring" to produce a flat rotation curve and the absence of conspicuous features marking the edge of the luminous component (Casertano \& van Gorkom 1991). However the conspiracy breaks down in the inner, bulge dominated region, which is always characterized by a steeply increasing rotation curve whose detailed shape and overall slope depends strongly on the galaxy luminosity and morphological type (Persic, Salucci \& Stel 1996; Sofue et al. 1999). For the galaxies in our sample, only for NGC 2841 the case could be made of a bulge-disk conspiracy: the rotation curve is approximatively flat all the way to the innermost point, $\sim 2 \mathrm{kpc}$ (Begeman 1987). In all other cases, the bulge contribution is insignificant by the time the flat part of the rotation curve is reached (Broeils 1992; Kent 1987). In other words there is no direct evidence in these galaxies of a coupling between bulge and halo. At most, a connection between the $v_{c}-\sigma_{c}$ relation and the diskhalo conspiracy can be thought of in the following terms. A rough correlation exists between maximum rotational velocity and Hubble type (e.g. Casertano \& van Gorkom 1991); coupled with the correlation between Hubble type and bulge luminosity and between the latter and the bulge velocity dispersion (Ko-

\footnotetext{
${ }^{2} \Omega_{\Lambda}=0.7, \Omega_{m}=0.3, h=0.7$
}

rmendy \& Illingworth 1983), a correlation between $v_{c}$ and $\sigma_{c}$ ensues. However, as in the case for the $M_{\bullet}-\sigma_{c}$ relation, which is "expected" given the $M_{\bullet}-L_{B}$ and the Faber-Jackson relation, the $v_{c}-\sigma_{c}$ relation is tighter, and therefore more fundamental, than any of the correlations mentioned above.

One last possibility to explore is whether the $v_{c}-\sigma_{c}$ relation could be nothing but the Tully-Fisher relation in disguise. Verheijen (2001) finds negligible intrinsic scatter for the $K$-band Tully-Fisher relation when the circular velocity in the flat part of the rotation curve is substituted to the maximum rotational velocity. The tightness of the relation implies a fundamental connection between DM halo mass and the total baryonic mass. The connection with bulge mass (and hence $\sigma_{c}$ ) is however not immediate (Norman, Sellwood \& Hasan 1996). For instance, the bulge to disk fraction could conceivably depend on the detailed form of the halo angular momentum profile (Bullock et al. 2001) and the details of the angular momentum exchange between gas clouds during dissipation (van den Bosch et al. 2002). It should be further noticed that Verheijen (2001) finds that the Tully-Fisher relation holds with negligible intrinsic scatter down to $v_{c} \sim 80 \mathrm{~km} \mathrm{~s}^{-1}$, while the $v_{c}-\sigma_{c}$ relation shown in Figure 3 seems to break down below $v_{c} \sim 150 \mathrm{~km}$ $\mathrm{s}^{-1}$.

\section{A RELATION BETWEEN SBHS AND DM HALOS}

Not being the result of a dynamical tautology, the near invariance of $\sigma_{c} / v_{c}$ over two orders of magnitude in $R\left(v_{c}\right) / R\left(\sigma_{c}\right)$ seems to indicate a remarkable uniformity in the (luminous + dark) mass density profile along the Hubble sequence. In particular, characterizing the slope and normalization of the relation might help in constraining theoretical models and numerical simulation (e.g. Steinmetz \& Muller 1995) following the formation and evolution of galaxies.

Furthermore, as discussed in $\$ 2$, the existence of the $v_{c}-\sigma_{c}$ relation is strongly suggestive of a tight correlation between M. and the total gravitational mass of the SBH host galaxy. If the rotation curves for all galaxies plotted in Fig. 3 are flat at a distance $R$ from the center, i.e. $v(R)=v_{c}$, the virial theorem ensures that $M(R) \propto v_{c}^{2}$. Equations (1) and (2) then imply $M_{\bullet} \propto M(R)^{2.7}$.

Several proposed scenarios trace the origin of SBHs to the very early stages of structure formation (Cattaneo, Haehnelt \& Rees 1999; Silk \& Rees 1998; Haehnelt \& Rees 1993; Umemura, Loeb \& Turner 1993). By defining the depth of the potential well, the mass and distribution of the DM halo control not only the formation of SBHs, but also their relationship to the luminous matter. Relating $V_{c}$ to $M_{D M}$ is no trivial task, but at least a rough attempt can be made as follows. In a CDM-dominated universe, $M_{D M}$ is uniquely determined by the halo velocity $v_{\text {vir }}$ measured at the virial radius, $R_{v i r}$. The latter is defined as the radius at which the mean density exceeds the mean universal density by a constant factor, generally referred to as the "virial overdensity" $\Delta_{v i r}$. Based on this definition, and in virtue of the virial theorem, it immediately follows that $M_{D M}$ must be proportional to the third power of $v_{v i r}$. Being a function of $\Delta_{v i r}$, the constant of proportionality depends on the adopted cosmology and may vary with time (e.g. Bryan \& Norman 1998; Navarro \& Steinmetz 2000). For the purpose of this paper, we will adopt the same $\Lambda$ CDM cosmological model used by Bullock et al. $(2001)^{2}$. Under these assumptions: 


$$
M_{D M}=2.7 \times 10^{12}\left(v_{\text {vir }} / 200 \mathrm{~km} \mathrm{~s}^{-1}\right)^{3} M_{\odot}
$$

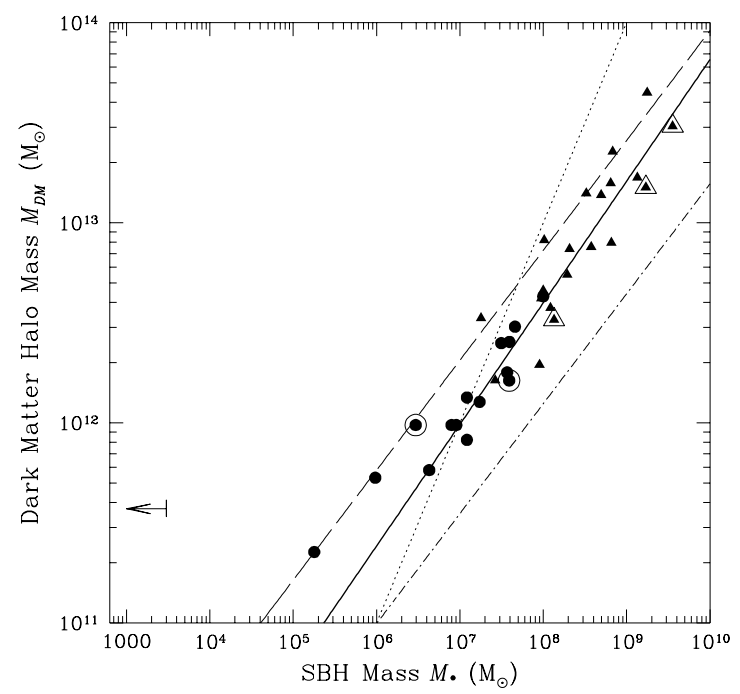

FIG. 5.- Same as Fig. 3, but with $\sigma_{c}$ transformed into SBH mass using equation (1), and $v_{c}$ transformed into DM halo mass following the prescriptions of Bullock et al. (2001). Solid circles are spirals, solid triangles ellipticals. Symbols accentuated by a larger open contour identify galaxies having a dynamical estimate of $M_{\bullet}$, which was used in the plot (Merritt \& Ferrarese 2002). The upper limit on the SBH mass in NGC 598 is marked by the arrow. The dotted line represents a constant ratio $M_{D M} / M_{\bullet}=10^{5}$. The solid line corresponds to the best fit using Bullock's et al. prescription to relate $v_{v i r}$ to $v_{c}$ (equation 5). The dashed line shows where the galaxies would lie if $v_{v i r}=v_{c}$ (equation 4), while if $v_{c} / v_{v i r}=1.8$, as proposed by Seljak (2002), the points would move to the location shown by the dot-dashed line (equation 7).

(Bullock et al. 2001).

In a zeroth order approximation, we might assume that $v_{v i r} \sim$ $v_{c}$. Combining equations (1), (2) and (3) gives:

$$
\frac{M_{\bullet}}{10^{8} M_{\odot}} \sim 0.027\left(\frac{M_{D M}}{10^{12} M_{\odot}}\right)^{1.82}
$$

However, for any reasonable shape of the DM density profile, the circular velocity will decrease towards $R_{v i r}$, in other words, $v_{c}$ as listed in Table 1 is most certainly an upper limit to $v_{v i r}$. The shape of the rotation curve, and therefore the $v_{c} / v_{v i r}$ ratio is of course strongly dependent on the assumed DM density profile. We will again follow Bullock et al. (2001) in adopting an NFW profile (Navarro, Frenk \& White 1995) characterized by an "inner" radius $r_{s}$. In this specific application, $v_{v i r} / V_{c}(R)$ is a function of two variables, the concentration parameter $C_{v i r}=R_{v i r} / r_{s}$ and the ratio $R / r_{s}$ (Bullock et al. 2001). N-body simulations (Bullock et al. 2001) show that for present day halos in the $10^{11}-10^{14} M_{\odot}$ range, $C_{v i r}$ depends weakly on $M_{D M}$ : $C_{v i r} \sim 5 \times 10^{2} M_{v i r}^{-0.13}$, where $M_{v i r}$ is in units of $M_{\odot}$. Therefore, for $R_{v i r} \sim$ a few $\times 10^{2} \mathrm{kpc}$ (Klypin et al. 2001), $r_{s}=R_{v i r} / C_{v i r} \sim$ a few to a few $\times 10 \mathrm{kpc}$, i.e. $r_{s}$ is likely sampled by the flat $\mathrm{HI}$ rotation curves used in deriving the $v_{c}-\sigma_{c}$ relation. This is a reasonable conclusion: in the region of space dominated by DM, the turnover in the rotation curve can be roughly expected to coincide with the turnover in the DM density profile. Therefore, by assuming $R=r_{s}, v_{v i r} / v_{c}(R)$ is a function of $M_{D M}$ only. By using equation (3) to express $v_{v i r}$ as a function of $M_{D M}$, Bullock's et al. results can be approximated as:

$$
\frac{M_{D M}}{10^{12} M_{\odot}} \sim 1.40\left(\frac{V_{c}}{200 \mathrm{~km} \mathrm{~s}^{-1}}\right)^{3.32} .
$$

By using equation (5) to transform $v_{c}$ to $M_{D M}$, and equation (1) to transform $\sigma_{c}$ to $M_{\bullet}$, equation (2) can be written as (Fig. 5):

$$
\frac{M_{\bullet}}{10^{8} M_{\odot}} \sim 0.10\left(\frac{M_{D M}}{10^{12} M_{\odot}}\right)^{1.65}
$$

A third, completely independent way of converting the $v_{c}-\sigma_{c}$ relation into a relation between SBH and DM halo masses is the following. Observational constraints on the mass density profiles of DM halos have been recently derived (Seljak 2002) by combining observations of galaxy-galaxy lensing with the Tully-Fisher and fundamental plane relations. For $L_{*}$ galaxies, the circular velocity at the virial radius decreases by a factor 1.8 from its peak value at the optical radius. This decline is steeper than predicted by Bullock's et al. (2001) numerical simulations, possibly due to the neglect, in the latter work, of the baryonic contribution to the rotation curves at optical radii (Seljak 2002).

By assuming $v_{v i r} / v_{c}(R) \sim 0.56^{3}$, the relation between $M_{D M}$ and $M_{\bullet}$ becomes:

$$
\frac{M_{\bullet}}{10^{8} M_{\odot}} \sim 0.67\left(\frac{M_{D M}}{10^{12} M_{\odot}}\right)^{1.82}
$$

Comparing equations (4), (6) and (7) can give a rough idea of how the functional dependence of $M_{D M}$ on $M_{\bullet}$ depends on the assumptions made for the DM density profile and the resulting rotation curve.

\subsection{The Significance and Scatter of the $M_{\bullet}-M_{D M}$ Relation}

The $M_{\bullet}-M_{D M}$ relation implies that the formation of SBHs is controlled, perhaps indirectly, by the properties of the DM halos in which they reside. Whether the connection between SBHs and DM halos is of a more fundamental nature than the one between SBHs and bulges, reflected in the $M_{\bullet}-\sigma_{c}$ relation, is unclear at this time. The $M_{\bullet}-\sigma_{c}$ relation implies a scatter of the order of $20-25 \%$ in $M_{\bullet}$ for $\sigma_{c}=70-300 \mathrm{~km} \mathrm{~s}^{-1}$. The scatter in the $M_{\bullet}-M_{D M}$ relation is difficult to determine, since the uncertainty in the transformation between $v_{c}$ and $M_{D M}$ is largely unknown. Bullock et al. (2001) estimate $\Delta \log \left(C_{v i r}\right)=0.18$. In the range $v_{c}=50-400 \mathrm{~km} \mathrm{~s}^{-1}$, this translates into a $5 \%$ to $7 \%$ error in $M_{D M}$ in equation (5), and a $9 \%$ to $12 \%$ error on $M_{\bullet}$ in equation (6). This indicates that, at least formally, the scatter in the $M_{\bullet}-M_{D M}$ relation could be a factor two smaller than the scatter in the $M_{\bullet}-\sigma_{c}$ relation for any given choice of DM density profile and cosmological parameters. Secure conclusions, however, will have to await an empirical characterization of the $M_{\bullet}-M_{D M}$ relation, with both $M_{\bullet}$ and $M_{D M}$ determined directly from observations.

Additional clues on how bulges and DM halos influence the formation of SBHs can be derived by studying bulgeless spirals. Such galaxies have been forcefully neglected in the present study, where we required the existence of a bulge velocity dispersion in order to derive $M_{\bullet}$. Of the galaxies in Table 1 , NGC598 is the one with the least conspicuous bulge ${ }^{4}$. IC342

\footnotetext{
${ }^{3}$ This is likely a lower limit to $v_{v i r} / v_{c}(R)$, since the peak circular velocity is $\geq v_{c}(R)$.
} 
and NGC 6503 are late type spirals with minimal bulges; like NGC598, they deviate significantly from the $v_{c}$ relation defined by the more massive systems. All of the galaxies with no or minimal bulge for which a rotation curve exists, including both dwarf systems and low surface brightness (LSB) galaxies, have circular velocities below $150 \mathrm{~km} \mathrm{~s}^{-1}$ (e.g. Blais-Oulette, Amram \& Carignan 2001; de Blok \& Bosma 2002). In particular, in the LSB compilation of van den Bosch et al. (2001) the only two galaxies with maximum circular velocity in excess of 150 $\mathrm{km} \mathrm{s}^{-1}$ are also the only galaxies with a significant bulge component. Therefore, it does seem that bulges, as well as SBHs, are always formed in halos with circular velocity in excess of $\sim 150 \mathrm{~km} \mathrm{~s}^{-1}$. In less massive halos, the interplay between halo, bulge and the central SBH remains to be investigated. For this purpose attempts to directly measure the mass of a putative SBH in bulgeless galaxies is of primary importance.

Whether equation (4), (6) or (7) is adopted, $M_{\bullet}$ does not depend linearly on $M_{D M}$. According to equation (6), the ratio between SBH and halo mass decreases from $\sim 2 \times 10^{-4}$ at $M_{D M}=10^{14} M_{\odot}$ to $\sim 10^{-5}$ at $M_{D M}=10^{12} M_{\odot}$, i.e. less massive halos are less efficient in forming SBHs. This tendency becomes more pronounced for halos with $M_{D M} \lesssim 5 \times 10^{11} M_{\odot}$ : as mentioned above, NGC 6503, NGC 3198 and NGC 598 lie above the relation defined by the more massive galaxies. It must be pointed out, however, that the $M_{\bullet}-\sigma_{c}$ relation is not defined for $M_{\bullet}<10^{6} M_{\odot}$, indeed we have no direct evidence of the existence of nuclear black holes in this mass range. If such SBHs exist, the recent report of an upper limit of only a few $10^{3} M_{\odot}$ for the BH in NGC 598 (Merritt, Ferrarese \& Joseph 2001; Gebhardt et al. 2001), indicates that they are not more massive than would be inferred by extrapolating the $M_{\bullet}-\sigma_{c}$ relation to the $\sigma_{c} \sim 20 \mathrm{~km} \mathrm{~s}^{-1}$ range. This excludes the possibility that NGC 6503 and NGC 3198 could be reconciled with the $M_{\bullet}-M_{D M}$ relation defined by the more massive galaxies.

These findings are in qualitative agreement with the formation scenario envisioned by Haehnelt, Natarajan \& Rees (1998) and Silk \& Rees (1998). In an attempt to match the DM halo mass function to the luminosity function of quasars, Haehnelt et al. noted that as the quasar lifetime is increased, a match can be obtained only at the expense of steepening the slope of the relation between $M_{\bullet}$ and $M_{D M}$. In their model, a linear relation between $M_{\bullet}$ and $M_{D M}$ implies a quasar lifetime $t_{Q S O} \sim 3 \times 10^{5}$ $\mathrm{yr}$, about a factor 100 shorter than favored by current observational constraints (e.g. Martini \& Weinberg 2001; Ferrarese 2002). If $t_{Q S O} \sim \mathrm{a}$ few $\times 10^{7} \mathrm{yr}$, the slope of the $M_{\bullet}-M_{D M}$ relation must be increased to $\sim 1.7$, in rather remarkable agreement with the results derived in this paper.

Furthermore, Haehnelt et al. propose that SBH formation takes place during the very early stages of galaxy formation. Gas gravitating in a DM halo is deposited at the center of the halo at a rate which is proportional to the third power of the halo circular velocity $v_{v i r}$. Stars more massive than $\sim 10^{6} M_{\odot}$ undergo post-Newtonian instabilities - and therefore collapse before they can ignite hydrogen; for $v_{v i r}$ larger than $\sim 200 \mathrm{~km}$ $\mathrm{s}^{-1}$, such stars can be formed on short enough timescales that the collapse will take place before the star has had a chance to burn all of its fuel. Only halos with $v_{v i r} \gtrsim 200 \mathrm{~km} \mathrm{~s}^{-1}$ (or masses $\gtrsim 10^{12} M_{\odot}$ ) would therefore host SBHs, and only SBHs with
$M_{\bullet}>10^{6} M_{\odot}$ can be formed in such halos. It is therefore possible that galaxies such as NGC 6503 and NGC 3198 do not contain SBHs.

\section{CONCLUSIONS}

The results in this paper can be summarized as follows:

- We have considered a sample of 15 spiral galaxies with published measurements of both the bulge velocity dispersion $\sigma_{c}$ and the disk circular velocity $v_{c}$, measured beyond the optical radius. The sample displays a tight correlation between the two quantities. Furthermore, in a $\log (\sigma)-\log \left(v_{c}\right)$ plane, the narrow strip occupied by the spirals blends smoothly with that occupied by a sample of 20 elliptical galaxies (from Kronawitter et al. 2001 and Gerhard et al. 2001). This is an interesting result, in view of the different methods used to derive $v_{c}$ for the two samples, and of the dissimilarities between the shapes of the rotation curves observed in spirals and modelled in elliptical galaxies.

- The ellipticals and spirals define a common, linear (in a $\log -\log$ plane) $v_{c}-\sigma_{c}$ relation for $70<\sigma_{c}<350 \mathrm{~km} \mathrm{~s}^{-1}$. Three galaxies have $\sigma_{c}$ below this range; all three deviate from the relation in the sense of having smaller than expected values of $\sigma_{c}$. The discrepancy is largest - a factor 7 larger than the standard deviation on $\sigma_{c}$ - for the galaxy with the smallest value of $\sigma_{c}$, NGC 598 .

- Rotation curves for all of the galaxies appear to be flat in the outer parts. In virtue of the $M_{\bullet}-\sigma_{c}$ relation and the virial theorem, the $v_{c}-\sigma_{c}$ relation must therefore imply that the masses of supermassive black holes correlate with the gravitational mass of their host galaxy, measured within a given radius $R>R_{25}$.

- If the further concession is made that, in CDM scenarios of structure formation, the virial mass of the DM halo is uniquely determined by $v_{c}$ (Navarro \& Steinmetz 2000, Bullock et al. 2001), the $v_{c}-\sigma_{c}$ relation is the observational evidence that SBH masses correlate tightly with the mass of the DM halos in which, presumably, they are formed. The relation between $M_{\bullet}$ and $M_{D M}$ is nonlinear: the ratio between $M_{\bullet}$ and $M_{D M}$ decreases with decreasing halo mass. This trend becomes more severe for $M_{D M} \lesssim 5 \times 10^{11} M_{\odot}$. It is possible that less massive halos might indeed be unable to form SBHs, in qualitative agreement with the theoretical arguments proposed by Haehnelt, Natarajan \& Rees (1998) and Silk \& Rees (1998).

I wish to thank Pat Côté, David Merritt, Milos Milosavljevic and Jerry Sellwood for providing the answers to many questions. Comments from Uros Seljak, James Bullock and the anonymous referee have also been of great help. This work is partly supported by NASA/LTSA grant NAG5-8693, and has made use of the NASA/IPAC Extragalactic Database (NED).

\footnotetext{
${ }^{4}$ The existence of a rarefied bulge in this galaxy is supported by both spectroscopic and photometric data (e.g. Minniti, Olszewki, \& Rieke 1993; Regan \& Vogel 1994; Mighell \& Rich 1995; Minniti 1996).
} 


\section{REFERENCES}

Adams, F.C., Graff, D.S., \& Richstone, D. 2000, ApJ, 551, L31

Akritas, M. G. \& Bershady, M. A. 1996, ApJ, 470, 706

Arnaboldi, M., et al. 1998, ApJ, 507, 759

Awaki, H., et al. 1994, PASJ, 46, L65

Baggett, W.E., Baggett, S.M., \& Anderson, K.S.J. 1998, AJ, 116, 1626

Begeman, K.G. 1987, PhD Thesis

Biermann, P.L., Kronberg, P.P., \& Schmutzler, T. 1989, 208, 22

Bosma, A. 1981, AJ, 86, 1825

Bottema R., 1988 A\&A 197, 105

Bottema, R. 1992, A\&A 257,69

Broeils 1992, PhD. Thesis

Bullock, J.S., et al. 2001, MNRAS, 321, 559

Casertano, S., \& van Gorkom, J.H. 1991, AJ, 101, 1231

Cattaneo, A., Haehnelt, M. G. \& Rees, M. J., 1999, MNRAS, 308, 77

Côté, P. et al. 2001, ApJ, 559, 828

Corbelli, E., \& Salucci, P. 2000, MNRAS, 311, 441

Davies, M. et al. 1987, ApJ, 64, 581

de Blok, W.J.G. \& Bosma, A. 2002, A\&A, 385, 816

de Blok, W.J.G., McGaugh, S.S. \& Rubin, V. 2001, AJ, 122, 2396

de Vaucouleurs, G., de Vaucouleurs, A., Corwin, H. G., Buta, R. J., Paturel, G.

\& Fouque, P. 1991, Third Reference Catalog of Bright Galaxies (New York:

Springer) (RC3)

Dressler, A. 1984, ApJ, 286, 97

Faber, S., \& Gallagher, J.S. 1979, ARAA, 17, 135

Fabricant, D., Rybicki, G., \& Gorenstein, P. 1984, ApJ, 286, 186

Ferrarese, L. 2002, to anear in High Energy Emission Around Black

Holes, eds. C.-H. Lee (astro-ph/0203047).

Ferrarese, L. \& Merritt, D. 2000, ApJ, 539, L9

Ferrarese, L., et al. 2000, ApJ, 529, 745

Gebhardt, K., et al. 2000, AJ, 119, 1157

Gebhardt, K., et al. 2001, AJ, 122, 2469

Gebhardt, K., et al. 2000, AJ, 119, 1157

Genzel, R., et al. 2000, astroph/0001428

Gerhard, O., Kronawitter, A., Saglia, R.P., \& Bender, R. 2001, AJ, 121, 1936

Graham, A.W., Erwin, P., Caon, N., \& Trujillo, I. 2001, ApJ, 563, L11

Haehnelt, M. G. \& Kauffmann, G., 2000, MNRAS, 318, L35

Haehnelt, M. G., Natarajan, P. \& Rees, M. J. 1998, MNRAS, 300, 817

Haehnelt, M. G., \& Rees, M.J. 1993, MNRAS, 263, 168

Irwin, J.A., \& Seaquist E.R. 1991, ApJ, 371, 111

Jorgensen, I., Franx, M. \& Kjaergaard, P. 1995, MNRAS, 276, 1341

Kent, S.M. 1987, in "Nearly Normal Galaxies: from the Plank Time to the

Present", eds. S.M. Faber (Springer, NY), p.81

Kent, S.M. 1989, PASP, 101, 489

Klypin, A. Zhao, H, \& Somerville, R.S, 2001, ApJ submitted (astro$\mathrm{ph} / 0110390)$

Koribalski, B., Dickey, J.M., \& Mebold, U. 1993, ApJ, 402, L41

Kormendy, J., \& Illingworth, G. 1982, ApJ, 256, 460

Kormendy, J., \& Illingworth, G. 1983, ApJ, 265, 632

Kormendy, J., \& Richstone, D., 1995, ARAA, 27, 235

Kronawitter, A., Saglia, R.P., Gerhard, O., \& Bender, R. 2000, A\&AS, 144, 53

Krumm, N., \& Salpeter, E.E. 1979, AJ, 84, 1138

Lauer, T. 1985, ApJ, 292, 292
Loeb, A., \& Rasio, F. 1994, ApJ, 432, L52

Martini, P., \& Weinberg, D.H. 2001, ApJ, 437, 12

Matsushita, K., et al. 1998, ApJ, 499, 13

McElroy, D.B. 1995, ApJS, 100, 105

Merritt, D., \& Tremblay, B. 1993, AJ, 106, 2229

Merritt, D. \& Ferrarese, L. 2001b, MNRAS, 320, L30

Merritt, D. \& Ferrarese, L. 2001a, ApJ, 547, 140

Merritt, D., Ferrarese, L. \& Joseph, C. 2001, Science, 293, 1116

Merritt, D., \& Ferrarese, L. 2002 to appear in The Central Kpc of Starbursts and

AGNs, eds. IH Knapen, JE Beckman, I Shlosman, \& T I Mahoney (astro$\mathrm{ph} / 0107134$

Monaco, P., Salucci, P., \& Danese, L. 2000, MNRAS, 311, 279

Napolitano, N.R,Arnaboldi, M., Freeman, K.C., \& Capaccioli, M. 2001,

A\&A, in press (astro-ph/0109112)

Navarro, J.F., Frenk, C.S., \& White, S.D.M 1995, MNRAS, 275, 56

Navarro, J.F., \& Steinmetz, M. 2000, ApJ, 538, 477

Newton, K. 1980, MNRAS, 191, 169

Norman, C.A., Sellwood, J.A., \& Hasan, H. 1996, ApJ, 462, 114

Olling, R.P., \& Merrifield, M.R. 1998, MNRAS, 297, 943

Persic, M, \& Salucci, P. 1995, ApJS, 99, 501

Persic, M., Salucci, P., \& Stel, F. 1996, MNRAS, 281, 27

Robinson, I. 1965, Quasi-Stellar Source and Gravitational Collapse,

University of Chicago press

Rogstad, D.H., \& Shostak, G.S. 1972, ApJ, 220, L37

Rots, A.H., \& Shane, W.W. 1975; A\&A, 45, 25

Rubin, V., Ford, W.K., \& Thonnard, N. 1978, ApJ, 225, L107

Rubin, V., Ford, W.K., \& Thonnard, N. 1980, ApJ, 238, 471

Rubin, V., Thonnard N., \& Ford W.K. 1977, ApJ, 217, L1

Rubin, V. et al. 1982, ApJ, 261, 439

Rubin, V. et al. 1985, ApJ, 289, 81

Rupen M.P. 1991, AJ, 102, 48

Saglia, R.P., Kronawitter, A., Gerhard, O., \& Bender, R. 2000, AJ, 119, 153

Saika, D.J., et al. 1980, MNRAS, 245, 397

Schechter, P.L. 1983, ApJS 52, 425

Seljak, U. 2002, MNRAS, in press (astro-ph/0201450)

Silk, J. \& Rees, M. J. 1998, A\&A, 331, L4

Sofue, Y. \& Rubin, V. 2001, ARA\&A, 39, 137

Sofue, Y. et al. 1999, ApJ, 523, 136

Steinmetz, M., \& Muller, E. 1995, MNRAS, 276, 549

Terlevich, E., Diaz, A. I., Terlevich, R. 1990, MNRAS 242, 271

Umemura, M., Loeb, A., \& Turner, E.L. 1993, ApJ, 419, 459

van Albada, T.S. 1980, A\&A, 90, 123

van den Marel, R. 1991, MNRAS, 253, 710

van den Bosch, F. 2000, ApJ, 530, 177

van den Bosch, F. et al. 2000, AJ, 119, 1579

van der Kruit, P.C. 1974, ApJ, 188, 3

Zhang, B., \& Wyse, R., 2000, MNRAS, 313, 310

Whitmore, B.C., Kirshner, R.P. 1981, ApJ 250, 43

Whitmore, B.C., Kirshner, R.P., Schechter, P. L. 1979, ApJ 234, 68

Whitmore, B.C., Rubin, V.C., Ford, W.K., 1984 ApJ 287, 66

Yu, Q., \& Tremaine, S. 2002, MNRAS, in press. 
Table 1. Galaxy Sample ${ }^{1}$

\begin{tabular}{|c|c|c|c|c|c|c|c|c|c|}
\hline Galaxy & $\begin{array}{c}\text { Hubble } \\
\text { Type }\end{array}$ & $\begin{array}{c}\text { Distance } \\
(\mathrm{Mpc})\end{array}$ & $\left(\begin{array}{c}V_{c} \\
\left(\mathrm{~km} \mathrm{~s}^{-1}\right)\end{array}\right.$ & $\begin{array}{l}R\left(\mathrm{v}_{c}\right) \\
(\mathrm{kpc})\end{array}$ & $\begin{array}{c}R_{25} \\
(\mathrm{kpc})\end{array}$ & $\left(\begin{array}{c}\sigma \\
\left(\mathrm{km} \mathrm{s}^{-1}\right)\end{array}\right.$ & $\begin{array}{c}\sigma_{c} \\
\left(\mathrm{~km} \mathrm{~s}^{-1}\right)\end{array}$ & $\begin{array}{l}\operatorname{Ref}^{2} \\
\left(v_{c}\right)\end{array}$ & $\begin{array}{l}\operatorname{Ref}^{3} \\
(\sigma)\end{array}$ \\
\hline \multicolumn{10}{|c|}{ Galaxies with HI Rotation Curves } \\
\hline MW & SbI-II & 0.008 & $180 \pm 20$ & 28.0 & 11.4 & $100 \pm 20$ & $100 \pm 20$ & 1 & 1 \\
\hline IC342 & $\mathrm{SAB}(\mathrm{rs}) \mathrm{cd}$ & 2.0 & $185 \pm 10$ & 10.0 & 14.0 & $77 \pm 12$ & $77 \pm 12$ & 2 & 2 \\
\hline N224 & $\mathrm{SA}(\mathrm{s}) \mathrm{b}$ & 0.670 & $240 \pm 20$ & 27.0 & 21.4 & $161 \pm 16$ & $146 \pm 15$ & 3 & 3 \\
\hline N598 & $\mathrm{SA}(\mathrm{s}) \mathrm{cd}$ & 0.85 & $135 \pm 13$ & 15.5 & 8.7 & $27 \pm 7$ & $27 \pm 7$ & 4 & 4 \\
\hline N801 & $\mathrm{Sc}$ & 79.2 & $216 \pm 9$ & 60.2 & 37.3 & $137 \pm 20$ & $144 \pm 27^{4}$ & 5 & 5 \\
\hline N2841 & $\mathrm{SA}(\mathrm{r}) \mathrm{b}$ & 18.0 & $281 \pm 10$ & 81.2 & 21.3 & $192 \pm 13$ & $179 \pm 12$ & 6 & 6 \\
\hline N2903 & $\mathrm{SB}(\mathrm{s}) \mathrm{d}$ & 6.4 & $180 \pm 4$ & 24.2 & 11.7 & $105 \pm 13$ & $106 \pm 13$ & 6 & 7 \\
\hline N2998 & $\mathrm{SAB}(\mathrm{rs}) \mathrm{c}$ & 67.4 & $198 \pm 5$ & 46.7 & 28.3 & $107 \pm 24$ & $113 \pm 30^{4}$ & 5 & 5 \\
\hline N3198 & $\mathrm{SB}(\mathrm{rs}) \mathrm{c}$ & 9.4 & $150 \pm 3$ & 46.1 & 17.8 & $65 \pm 10$ & $69 \pm 13^{4}$ & 6 & 6 \\
\hline N4258 & $\mathrm{SAB}(\mathrm{s}) \mathrm{bc}$ & 6.6 & $210 \pm 20$ & 30.5 & 19.5 & $146 \pm 19$ & $138 \pm 18$ & 7 & 1 \\
\hline N4565 & $\mathrm{SA}(\mathrm{s}) \mathrm{b}$ & 17.1 & $264 \pm 8$ & 51.2 & 39.4 & $151 \pm 13$ & $151 \pm 13$ & 8 & 8 \\
\hline N5033 & $\mathrm{SA}(\mathrm{s}) \mathrm{c}$ & 11.9 & $195 \pm 5$ & 35.5 & 18.5 & $133 \pm 10$ & $122 \pm 9$ & 6 & 7 \\
\hline N5055 & $\mathrm{SA}(\mathrm{rs}) \mathrm{bc}$ & 8.0 & $180 \pm 5$ & 44.0 & 18.5 & $115 \pm 7$ & $103 \pm 6$ & 9 & 7 \\
\hline N6503 & $\mathrm{SA}(\mathrm{s}) \mathrm{cd}$ & 5.9 & $116 \pm 2$ & 11.4 & 6.1 & $45 \pm 7$ & $48 \pm 10^{4}$ & 6 & 9 \\
\hline N7331 & $\mathrm{SA}(\mathrm{s}) \mathrm{b}$ & 14.9 & $239 \pm 5$ & 37.3 & 46.0 & $150 \pm 14$ & $139 \pm 14$ & 6 & 7 \\
\hline \multicolumn{10}{|c|}{ Galaxies with Optical Rotation Curves } \\
\hline IC724 & $\mathrm{Sa}$ & 103.7 & $302 \pm 3$ & 31 & 35.3 & $229 \pm 17$ & $243 \pm 18^{4}$ & 10 & 3 \\
\hline N753 & $\mathrm{SAB}(\mathrm{rs}) \mathrm{bc}$ & 90.0 & $210 \pm 7$ & 21 & 32.9 & $129 \pm 18$ & $121 \pm 17$ & 11 & 3 \\
\hline N1353 & $\mathrm{SA}(\mathrm{rs}) \mathrm{bc}$ & 27.0 & $191 \pm 6$ & 5 & 13.3 & $85 \pm 11$ & $92 \pm 12$ & 12 & 3 \\
\hline N1357 & $\mathrm{SA}(\mathrm{s}) \mathrm{ab}$ & 26.2 & $259 \pm 5$ & 9 & 10.7 & $121 \pm 14$ & $121 \pm 14$ & 10 & 3 \\
\hline N1417 & $\mathrm{SA}(\mathrm{r}) \mathrm{b}$ & 54.2 & $240 \pm 9$ & 14.5 & 21.2 & $148 \pm 18$ & $148 \pm 18$ & 13 & 10 \\
\hline N1620 & $\mathrm{SAB}(\mathrm{rs}) \mathrm{bc}$ & 60.7 & $250 \pm 4$ & 20 & 25.5 & $117 \pm 19$ & $123 \pm 20^{4}$ & 12 & 3 \\
\hline N2639 & (R)SA(r)A: & 66.5 & $318 \pm 4$ & 12 & 17.6 & $193 \pm 13$ & $195 \pm 13$ & 10 & 3 \\
\hline $\mathrm{N} 2742$ & $\mathrm{SA}(\mathrm{s}) \mathrm{c}$ & 24.2 & $173 \pm 4$ & 9 & 10.6 & $68 \pm 30$ & $63 \pm 28$ & 11 & 7 \\
\hline $\mathrm{N} 2775$ & $\mathrm{SA}(\mathrm{r}) \mathrm{ab}$ & 24.3 & $270 \pm 4$ & 10 & 15.1 & $170 \pm 14$ & $162 \pm 13$ & 10 & 3 \\
\hline N2815 & $(\mathrm{R}) \mathrm{SB}(\mathrm{r}) \mathrm{b}$ & 40.0 & $278 \pm 3$ & 18 & 20.2 & $199 \pm 20$ & $199 \pm 20$ & 12 & 11 \\
\hline N2844 & $\mathrm{SA}(\mathrm{r}) \mathrm{a}:$ & 26.4 & $171 \pm 10$ & 6 & 5.9 & $114 \pm 12$ & $113 \pm 12$ & 10 & 3 \\
\hline N3067 & $\mathrm{SAB}(\mathrm{s}) \mathrm{ab}$ & 25.1 & $148 \pm 1$ & 6 & 8.9 & $82 \pm 28$ & $84 \pm 29^{4}$ & 12 & 10 \\
\hline N3145 & $\mathrm{SB}(\mathrm{rs}) \mathrm{bc}$ & 61.0 & $261 \pm 3$ & 23 & 27.4 & $161 \pm 12$ & $166 \pm 12$ & 12 & 3 \\
\hline N3223 & $\mathrm{SA}(\mathrm{r}) \mathrm{bc}$ & 46.5 & $261 \pm 11$ & 21 & 27.5 & $157 \pm 16$ & $163 \pm 17^{4}$ & 12 & 3 \\
\hline N3593 & $\mathrm{SA}(\mathrm{s}) 0 / \mathrm{a}$ & 12.4 & $101 \pm 4$ & 2.1 & 9.5 & $56 \pm 15$ & $56 \pm 15$ & 10 & 3 \\
\hline N4062 & $\mathrm{SA}(\mathrm{s}) \mathrm{c}$ & 13.1 & $154 \pm 13$ & 9 & 7.7 & $97 \pm 8$ & $90 \pm 7$ & 11 & 12 \\
\hline $\mathrm{N} 4321$ & $\mathrm{SAB}(\mathrm{s}) \mathrm{bc}$ & 15.1 & $216 \pm 6$ & 10 & 16.3 & $83 \pm 12$ & $83 \pm 12$ & 11 & 3 \\
\hline N4378 & (R) $\mathrm{SA}(\mathrm{s}) \mathrm{a}$ & 43.1 & $308 \pm 1$ & 9 & 18.1 & $206 \pm 19$ & $198 \pm 18$ & 10 & 3 \\
\hline N4448 & $\mathrm{SB}(\mathrm{r}) \mathrm{ab}$ & 16.8 & $187 \pm 3$ & 6 & 9.5 & $179 \pm 21$ & $175 \pm 21$ & 12 & 13 \\
\hline N4698 & $\mathrm{SA}(\mathrm{s}) \mathrm{ab}$ & 20.0 & $252 \pm 5$ & 8.2 & 11.6 & $134 \pm 19$ & $131 \pm 19$ & 10 & 10 \\
\hline N7217 & (R)SA(r)ab & 21.9 & $241 \pm 4$ & 9 & 12.4 & $185 \pm 18$ & $171 \pm 17$ & 12 & 13 \\
\hline N7541 & $\mathrm{SB}(\mathrm{rs}) \mathrm{bc}$ & 51.0 & $179 \pm 1$ & 12 & 25.7 & $104 \pm 14$ & $109 \pm 15^{4}$ & 11 & 3 \\
\hline N7606 & $\mathrm{SA}(\mathrm{s}) \mathrm{b}$ & 42.1 & $240 \pm 4$ & 30 & 32.9 & $136 \pm 23$ & $124 \pm 21^{4}$ & 12 & 10 \\
\hline
\end{tabular}

${ }^{1}$ Columns are: 1) Galaxy name; 2) Revised Hubble type; 3) Galaxy distance, from Ferrarese et al. 1999 or derived from the galaxy systemic velocity and $H_{0}=75 \mathrm{~km} \mathrm{~s}^{-1} \mathrm{Mpc}^{-1}$;4) Circular velocity measured at the outermost radius (col. 5) of the rotation curve, with references given in col. 9; 6) Galaxy diameter at the $B=25$ mag $\operatorname{arcsec}^{-2}$ isophote (from the RC3, de Vaucouleurs et al. 1991); 7) bulge velocity dispersion, with references given in col. $10 ; 8)$ bulge velocity dispersion corrected to an aperture of size $r_{e} / 8$.

${ }^{2}$ References for $v_{c}$ : 1. Olling \& Merrifield 1998 2. Newton 1980; 3. Kent 1989; 4. Corbelli \& Salucci $2000 ; 5$. Broeils 1992; 6. Begeman 1987; 7. van Albada 1980; 8. Krumm \& Salpeter 1979; 9. Bosma 1981; 10. Rubin et al. 1985; 11. Rubin, Ford \& Thonnard 1980; 12. Rubin, et al. 1982; 13. Persic \& Salucci 1995.

${ }^{3}$ Reference for $\sigma: 1$. Merritt, \& Ferrarese 2000; 2. Terlevich et al. 1990; 3. Whitmore \& Kirshner 1981; 4. Merritt et al. 2001 5. Schechter 1983; 6. Bottema 1988; 7. Heraudean \& Simien 1998; 8. Kormendy \& Illingworth 1982; 9. Bottema 1989; 10. Heraudeau et al. 1999; 11. Bottema, R. 1992; 12. Whitmore et al. 1984; 13. Whitmore et al. 1979 .

${ }^{4}$ No measurement of the bulge effective radius $R_{e}$ is available. The correction to the bulge velocity dispersion was calculated by assuming a ratio between $r_{e}$ and $R_{25}$ equal to the mean ratio calculated from the Baggett et al. (1998) for galaxies of the same Hubble type. 
TABLE 2

RESUlTS OF THE LINEAR REGRESSION FITS: $\boldsymbol{v}_{c}=\alpha \sigma_{c}+\beta$

\begin{tabular}{lcrrl}
\hline \hline \multicolumn{1}{c}{ Sample $^{(2)}$} & $N$ & $\alpha$ & $\beta$ & \multicolumn{1}{c}{$\chi_{r}^{2}$} \\
\hline 1. All spirals & 37 & $0.781 \pm 0.061$ & $0.70 \pm 0.13$ & 1.9 \\
2. Spirals, $R\left(v_{c}\right) / R_{25}>1.0$ only & 15 & $0.683 \pm 0.060$ & $0.89 \pm 0.12$ & 0.70 \\
3. Spirals, $R\left(v_{c}\right) / R_{25}>1.0$ and & 13 & $0.839 \pm 0.089$ & $0.55 \pm 0.19$ & 0.38 \\
$\quad$ excluding N3198 and N6503 & & & & \\
4. All Ellipticals & 20 & $0.94 \pm 0.11$ & $0.31 \pm 0.26$ & 0.66 \\
5. Samples 3 and 4 combined & 33 & $0.892 \pm 0.041$ & $0.44 \pm 0.09$ & 0.51 \\
\hline
\end{tabular}

${ }^{1}$ Units for $\sigma_{c}$ and $v_{c}$ are $\mathrm{km} \mathrm{s}^{-1}$.

${ }^{2} \mathrm{M} 33$ is excluded from all fits. 